\title{
Bounds on the Superconducting Transition Temperature: Applications to Twisted Bilayer Graphene and Cold Atoms
}

\author{
Tamaghna Hazra, Nishchhal Verma, and Mohit Randeria* \\ Department of Physics, The Ohio State University, Columbus, Ohio 43210, USA
}

(Received 13 June 2019; published 17 September 2019)

\begin{abstract}
Understanding the material parameters that control the superconducting transition temperature $T_{c}$ is a problem of fundamental importance. In many novel superconductors phase fluctuations determine $T_{c}$, rather than the collapse of the pairing amplitude. We derive rigorous upper bounds on the superfluid phase stiffness for multiband systems, valid in any dimension. This in turn leads to an upper bound on $T_{c}$ in two dimensions, which holds irrespective of pairing mechanism, interaction strength, or order-parameter symmetry. Our bound is particularly useful for the strongly correlated regime of low-density and narrowband systems, where mean-field theory fails. For a simple parabolic band in 2D with Fermi energy $E_{F}$, we find that $k_{B} T_{c} \leq E_{F} / 8$, an exact result that has direct implications for the 2D BCS-BEC crossover in ultracold Fermi gases. Applying our multiband bound to magic-angle twisted bilayer graphene, we find that band structure results constrain the maximum $T_{c}$ to be close to the experimentally observed value. Finally, we discuss the question of deriving rigorous upper bounds on $T_{c}$ in $3 \mathrm{D}$.
\end{abstract}

DOI: 10.1103/PhysRevX.9.031049

\section{INTRODUCTION}

Our work is motivated by the fundamental question, what limits the superconducting (SC) transition temperature $T_{c}$ ? Within BCS mean-field theory, and its extensions like Eliashberg theory, the amplitude of the SC order parameter is destroyed by the breaking of pairs, and $T_{c}$ scales with the pairing gap $\Delta$. The material parameters that control the mean-field $T_{c}$ are the electronic density of states (DOS) at the chemical potential $N(0)$ and the effective interaction, determined by the spectrum of fluctuations that mediate pairing.

Beginning with the pioneering experiments of Uemura [1] and theoretical ideas of Emery and Kivelson [2] on underdoped cuprates, it became clear that the mean-field picture of $T_{c}$ scaling with the pairing gap is simply not valid in many novel superconductors. The loss of SC order is then governed by fluctuations of the phase of the order parameter, rather than the suppression of its amplitude, and $T_{c}$ is related to the superfluid stiffness $D_{s}$. The material parameters that determine $D_{s}$ are rather different from those that determine the pairing gap $\Delta$.

\footnotetext{
* Corresponding author. randeria.1@osu.edu

Published by the American Physical Society under the terms of the Creative Commons Attribution 4.0 International license. Further distribution of this work must maintain attribution to the author(s) and the published article's title, journal citation, and DOI.
}

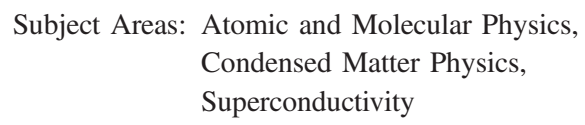

The question of mean-field amplitude collapse versus phase fluctuation dominated SC transition is brought into sharp focus by a variety of recent experiments in narrowband and low-density systems. One of the most exciting recent developments is the observation of very narrow bands in magic-angle twisted bilayer graphene (MA TBG) leading to correlation-induced "Mott" insulating states [3] and superconductivity [4] in their vicinity. Flatbands are also also expected to arise in various topological states of matter; see, e.g., Refs. [5-8]. BCS theory-based intuition suggests that narrow bands have a large DOS $N(0)$ and lead to high-temperature superconductivity. Is this true, or do phase fluctuations limit the $T_{c}$ ?

The extensive compilation of data in Fig. 6 of Ref. [4] suggests that all known superconductors have a $T_{c}$ that scales at most like a constant times the "Fermi energy $E_{F}$," though there is considerable leeway in defining $E_{F}$ in strongly correlated and multiband materials. We also note that ultracold Fermi gases in the strongly interacting regime of the BCS-BEC crossover $[9,10]$ exhibit experimental values [11] of $k_{B} T_{c} / E_{F}$ larger than those observed in the solid state. All of these observations raise the question of ultimate limits on the $T_{c}$ of a superconductor or paired superfluid.

In this paper, we obtain sharp answers to these questions, especially in 2D. First, we derive an upper bound on the superfluid stiffness $D_{s}(T) \leq \tilde{D}(T)$, where $\tilde{D}$ is proportional to the optical conductivity sum rule. This inequality is valid in all dimensions and for arbitrary interactions. 
We then use the Berezinskii-Kosterlitz-Thouless (BKT) theory in $2 \mathrm{D}$ to obtain $k_{B} T_{c} \leq \pi \tilde{D}\left(T_{c}\right) / 2$.

While the bound on $T_{c}$ is of completely general validity, it is most useful in the strongly correlated regime of narrowband and low-density systems, precisely where conventional mean-field approaches fail. We show that $\tilde{D}$ is necessarily "small" in such systems, and, in many cases of interest, $\tilde{D}$ is essentially determined by the (noninteracting) band structure.

We give several examples that illustrate the usefulness of our bounds for a variety of systems. For a single parabolic band we show that $k_{B} T_{c} \leq E_{F} / 8$ in $2 \mathrm{D}$. This exact result poses stringent constraints on the $T_{c}$ of the 2D BCS-BEC crossover in ultracold atoms. We also describe bounds on $T_{c}$ for the 2D attractive Hubbard model, relevant for current optical lattice experiments [12], that demonstrate the tension between breaking of pairs and phase fluctuations, and highlight the connection with a pairing pseudogap $[13,14]$.

Turning to multiband systems, we use available band structure results [15-19] for MA TBG to calculate $\tilde{D}$ and thus constrain its $T_{c}$ without any assumptions about the pairing mechanism or order-parameter symmetry. We obtain a rigorous (but weak) bound of $\simeq 15 \mathrm{~K}$. Using physically motivated approximations, we estimate a bound on $T_{c}$ as low as $6 \mathrm{~K}$.

Finally, we discuss the question of deriving similar bounds in 3D. We show that the presence of nonuniversal prefactors in the relation between $T_{c}$ and $D_{s}$, as well their scaling behavior near a SC quantum critical point, poses challenges in deriving a rigorous bound in $3 \mathrm{D}$.

\section{RESULTS}

We first outline our main results and then give a detailed derivation and specific applications. We consider a Fermi system described by the general Hamiltonian,

$$
\mathcal{H}=\mathcal{H}_{K}+\mathcal{H}_{\text {int }}, \quad \mathcal{H}_{K}=\sum_{\mathbf{k}, m, \sigma} \epsilon_{m}(\mathbf{k}) c_{\mathbf{k} m \sigma}^{\dagger} c_{\mathbf{k} m \sigma},
$$

where $\mathbf{k}$ is crystal momentum, $m$ is a band label, and $\sigma$ the spin. $\mathcal{H}_{K}$ is the kinetic energy and $\mathcal{H}_{\text {int }}$ describes interactions (electron-phonon, electron-electron, etc.), including those that give rise to superconductivity. The external vector potential A enters $\mathcal{H}$ through a Peierls substitution in the tight-binding representation of $\mathcal{H}_{K}$, but does not affect $\mathcal{H}_{\text {int }}$. For now, we ignore disorder and return to it at the end of the paper.

The macroscopic superfluid stiffness $D_{s}$ determines the free-energy cost of distorting the phase of the SC order parameter $|\Delta| e^{i \theta}$ via the Boltzmann factor $\exp \left(-D_{s} \int d^{d} \mathbf{r}|\nabla \theta|^{2} \mid / 2 k_{B} T\right)$. It is related to the London penetration depth via $1 / \lambda_{L}^{2}=\left(4 \mu_{0} e^{2} / \hbar^{2}\right) D_{s}$ in 3D. Microscopically, $D_{s}$ can be calculated as the static, long-wavelength limit of the transverse current response $[20,21]$ to a vector potential. (Our results are equally valid for neutral superfluids with rotation playing the role of the magnetic field.) We obtain a rigorous upper bound valid in any dimension:

$$
D_{s}(T) \leq \tilde{D}(T)=\frac{\hbar^{2}}{4 \Omega} \sum_{\mathbf{k}, m m^{\prime}, \sigma} M_{m m^{\prime}}^{-1}(\mathbf{k})\left\langle c_{\mathbf{k} m \sigma}^{\dagger} c_{\mathbf{k} m^{\prime} \sigma}\right\rangle,
$$

where $\Omega$ is the volume of the system and $M_{m m^{\prime}}^{-1}(\mathbf{k})$ is an inverse mass tensor that depends only on the electronic structure of $\mathcal{H}_{K}$; see Eq. (5). The temperature and interactions impact $\tilde{D}$ only through $\left\langle c_{\mathbf{k} m \sigma}^{\dagger} c_{\mathbf{k} m^{\prime} \sigma}\right\rangle$, where the thermal average is calculated using the full $\mathcal{H}$.

We next use $D_{s}$ to provide an upper bound on the SC transition temperature in 2D. We use the Nelson-Kosterlitz [22] universal relation to obtain

$$
k_{B} T_{c} \leq \pi \tilde{D}\left(T_{c}\right) / 2
$$

For a weak-coupling superconductor, $T_{c}$ is well described by mean-field theory, and our result, though valid as an upper bound, may not be very useful. On the other hand, as we show below, for a strongly interacting system the bound gives insight both into the value of $T_{c}$ and on its dependence on parameters.

\section{BOUND ON SUPERFLUID STIFFNESS}

The intuitive idea behind $D_{s} \leq \tilde{D}$ is as follows. $\left(2 \pi e^{2} / \hbar^{2}\right) \tilde{D}=\int_{0}^{\infty} d \omega \operatorname{Re} \sigma(\omega)$ is the optical conductivity spectral weight integrated over the bands in Eq. (1), and $\left(4 \pi e^{2} / \hbar^{2}\right) D_{s}$ is the coefficient of the $\delta(\omega)$ piece in $\operatorname{Re} \sigma(\omega)$ in the SC state; note that $\int_{0}^{\infty} d \omega \delta(\omega)=1 / 2$. The inequality Eq. (2) says that the weight in the SC delta function must be less than or equal to the total spectral weight.

To derive Eq. (2), we use the Kubo formula for $D_{s}$ as a linear response $[20,21]$ to an external vector potential in an arbitrary direction $a$,

$$
D_{s}=\tilde{D}-\left(\hbar^{2} / 4 e^{2}\right) \chi_{j_{a} j_{a}}^{\perp}(\mathbf{q} \rightarrow 0, \omega=0),
$$

where $\tilde{D}$ is the diamagnetic response $\sim\left\langle\delta^{2} \mathcal{H} / \delta A_{a}{ }^{2}\right\rangle$, while $\chi^{\perp}$ is the transverse current-current correlation function. $\tilde{D}$ is given by Eq. (2) with

$$
M_{m m^{\prime}}^{-1}(\mathbf{k})=\sum_{\alpha \beta} U_{m, \alpha}^{\dagger}(\mathbf{k}) \frac{\partial^{2} t_{\alpha \beta}(\mathbf{k})}{\partial\left(\hbar k_{a}\right)^{2}} U_{\beta, m^{\prime}}(\mathbf{k}) .
$$

Here $\alpha, \beta$ label orbitals or sites within a unit cell of a Bravais lattice, $t_{\alpha \beta}(\mathbf{k})$ is the Fourier transform of the hopping $t_{\alpha \beta}\left(\mathbf{r}_{i \alpha}-\mathbf{r}_{j \alpha}\right)$, and $U_{\alpha, m}(\mathbf{k})$ is the unitary transformation that diagonalizes $t_{\alpha \beta}(\mathbf{k})$ to the band basis 
$\epsilon_{m}(\mathbf{k}) \delta_{m, m^{\prime}}$. The inverse mass tensor in Eq. (5) also depends on the direction $a=x, y, \ldots$ through the derivative with respect to $k_{a}$ on the right-hand side; however, we do not show this $a$ dependence explicitly to simplify the notation. These results are derived in Appendix A, and the relation to the optical sum rule shown in Appendix B; see also Ref. [23].

We next turn to the second term in Eq. (4). From its Lehmann representation we see that $\chi^{\perp}(\mathbf{q} \rightarrow 0, \omega=0) \geq 0$ at all temperatures; see Appendix $\mathrm{C}$. We thus obtain $D_{s}(T) \leq \tilde{D}(T)$.

For a single band system Eqs. (2) and (5) simplify greatly and we get $\tilde{D}=(4 \Omega)^{-1} \sum_{\mathbf{k}, \sigma}\left(\partial^{2} \epsilon(\mathbf{k}) / \partial k_{a}^{2}\right) n_{\sigma}(\mathbf{k})$, where the momentum distribution $n_{\sigma}(\mathbf{k})=\left\langle c_{\mathbf{k} \sigma}^{\dagger} c_{\mathbf{k} \sigma}\right\rangle$. This allows us to recover well-known special cases. (1) With nearestneighbor (NN) hopping on a square or cubic lattice, $\partial^{2} \epsilon(\mathbf{k}) / \partial k_{a}^{2} \sim \epsilon(\mathbf{k})$, and $\tilde{D}$ is proportional to the kinetic energy. (2) A parabolic dispersion $\epsilon(\mathbf{k})=\hbar^{2} k^{2} / 2 m$ leads to the simple result $\tilde{D}=\hbar^{2} n / 4 m$, independent of $T$ and of interactions. Here $D_{s}(T)=\hbar^{2} n_{s}(T) / 4 m$, and our bound simply says that the superfluid density $n_{s}(T) \leq n$ the total density.

For materials with nonparabolic dispersion and/or multiple bands, $\tilde{D}$ depends on $T$ and interactions. It is thus illuminating to derive a bound for $\tilde{D}$ which depends only on the density. We describe the single-band result here, relegating the multiband generalization to Appendix $\mathrm{D}$. We write $\mathcal{H}_{K}=-\sum_{\mathbf{R} \delta \sigma}\left[t(\boldsymbol{\delta}) c_{\mathbf{R}+\delta, \sigma}^{\dagger} c_{\mathbf{R}, \sigma}+\right.$ H.c. $]$ with translationally invariant hopping amplitudes $t(\boldsymbol{\delta})$ that depend only the vector $\boldsymbol{\delta}$ connecting lattice sites $\mathbf{R}$ and $\mathbf{R}+\boldsymbol{\delta}$. We couple the system to a vector potential and compute $\tilde{D}$, which involves terms like $\sum_{i, j} \delta_{a}^{2} t(\boldsymbol{\delta})\left\langle c_{i}^{\dagger} c_{j}\right\rangle$ with $\boldsymbol{\delta}=i-j$ (schematically). We note that $\tilde{D} \geq 0$, since it is the sum rule for $\operatorname{Re} \sigma(\omega) \geq 0$. We then use the triangle inequality and the Cauchy-Schwarz inequality $\left|\left\langle c_{i}^{\dagger} c_{j}\right\rangle\right| \leq \sqrt{\left\langle n_{i}\right\rangle\left\langle n_{j}\right\rangle}=n$ to obtain $D_{s} \leq \tilde{D} \leq n \sum_{\delta} \delta_{a}^{2}|t(\boldsymbol{\delta})| / 2$. This shows that for small hopping and/or low density, one necessarily has a small $D_{s}$.

\section{IV. $T_{c}$ BOUND IN 2D}

For a BKT transition in 2D, the $T_{c}$ and the stiffness $D_{s}$ are related by the universal ratio [22] $k_{B} T_{c} / D_{s}\left(T_{c}^{-}\right)=\pi / 2$. Together with Eq. (2), $D_{s}\left(T_{c}^{-}\right) \leq \tilde{D}\left(T_{c}\right)$, we then immediately obtain Eq. (3). In an anisotropic system $\tilde{D}$ depends on $a=x, y$ through the $\partial^{2} / \partial k_{a}^{2}$ in Eq. (5). We can use $\tilde{D}=\max \left\{\tilde{D}_{x}, \tilde{D}_{y}\right\}$ to obtain a bound on $T_{c}$; however, we argue in Appendix $\mathrm{H}$ for a much stronger result $\tilde{D}=\left[\tilde{D}_{x} \tilde{D}_{y}\right]^{1 / 2}$ in $2 \mathrm{D}$.

We emphasize that Eq. (3) with $\tilde{D}\left(T_{c}\right)$ on the rhs is sufficient to derive the rigorous results below. However, to obtain the intuitively more appealing result, $k_{B} T_{c} \leq$ $\pi \tilde{D}(0) / 2$, we need to assume that $D_{s}(T)$ is a decreasing function of $T$, so that $D_{s}\left(T_{c}^{-}\right) \leq D_{s}(0) \leq \tilde{D}(0)$.

\section{2D PARABOLIC DISPERSION}

Consider a single band with $\epsilon(\mathbf{k})=\hbar^{2} k^{2} / 2 m$ with density $n$, so that the Fermi energy $E_{F}=\pi \hbar^{2} n / m$, and arbitrary interactions that lead to pairing and superconductivity. Then $M^{-1}(\mathbf{k})=m^{-1}$ and $\Omega^{-1} \sum_{\mathbf{k}, \sigma} n_{\sigma}(\mathbf{k} ; T)=n$ independent of $T$ and interactions, so that $\tilde{D}=\hbar^{2} n / 4 m$. Equation (3) then leads to the simple result

$$
k_{B} T_{c} \leq E_{F} / 8,
$$

which must be obeyed independent of the strength of attraction or order-parameter symmetry, provided the system exhibits a BKT transition. In a weak-coupling superconductor, $T_{c}$ will actually be much smaller than $E_{F} / 8$ but, as we discuss next, the bound can be saturated in systems with strong interactions, such as the 2D BCS-BEC crossover experiments in ultracold Fermi gases.

\section{2D BCS-BEC CROSSOVER}

In ultracold Fermi gas experiments the two-body $s$-wave interaction between atoms is tuned using a Feshbach resonance. This has led to deep insights into the crossover $[9,10]$ from the weak-coupling BCS limit with large Cooper pairs all the way to the BEC of tightly bound diatomic molecules. Asymptotically exact results are available in both the BCS and BEC limits; however, the crossover regime between the two extremes is very strongly interacting, with pair size comparable to the interparticle spacing, and is much less understood. It is precisely here that our exact upper bound Eq. (6) is relevant.

The $2 \mathrm{D}$ crossover for $s$-wave pairing is parametrized by the dimensionless interaction [24] $\log \left(E_{b} / E_{F}\right)$, where $E_{b}$ is the binding energy of the two-body bound state in vacuum and $E_{F}$ the Fermi energy. In the weak-coupling BCS limit $\left(E_{b} \ll E_{F}\right)$, the mean field $k_{B} T_{c} \sim \sqrt{E_{F} E_{b}}$ [24], with a prefactor that has been computed including the GorkovMelik-Barkhudarov correction $[25,26]$. Clearly $T_{c}$ is much smaller than our bound.

In the BEC limit $\left(E_{b} \gg E_{F}\right)$ the composite bosons have mass $2 m$, density $n / 2$, and an inter-boson scattering length $a_{b}$, where $E_{b} / E_{F} \sim 1 / n a_{b}^{2}$ [26]. The 2D dilute Bose gas has $k_{B} T_{c}=E_{F} /\left[2 \log \log \left(2 / n a_{b}^{2}\right)\right]$ [27], which is valid in the regime $\log \log \gg 1$. This too is smaller than our bound, though our exact result cautions against a naive extrapolation of the BEC limit result into the strong interaction regime.

The results of the 2D Fermi gas experiment of Ref. [28] seem to violate Eq. (6) in the crossover regime. We note, however, that our bound is obtained for a strictly 2D system in the thermodynamic limit, while the experiment is on a quasi-2D system in a harmonic trap, from which it is difficult to accurately determine the BKT $T_{c}$. The finite size of the trap raises $T_{c}$; even the noninteracting Bose gas in a 2D harmonic trap has a nonzero $T_{c}$. 


\section{MAGIC-ANGLE TWISTED BILAYER GRAPHENE}

Let us next turn to a multiband system of great current interest. The existence of very narrow bands in MA TBG was predicted by continuum electronic structure calculations $[15,16]$ that pointed out the crucial role of $\alpha=w / \hbar v_{F}^{0} K \theta$, where $\theta$ is the twist angle between the two layers, $w$ is the interlayer tunneling, $v_{F}^{0}$ the bare Fermi velocity, and $K$ the Dirac-node location in monolayer graphene. It was predicted that $v_{F}$ in TBG can be tuned to zero [15], with a bandwidth less than $10 \mathrm{meV}$ by choosing certain magic angles $\theta$, the largest of which, $\approx 1.1^{\circ}$, has now been achieved in experiments $[3,4]$. Recently, pressure tuning of $w$ has also resulted in very narrow bands [29].

Little is known at this time about the nature of the SC state or the pairing mechanism, though the observed nonlinear $I-V$ characteristics $[3,4]$ are consistent with a BKT transition. Proximity to a Mott insulator and narrow bandwidth suggest the importance of electron correlations, while the extreme sensitivity of the dispersion to structure suggests that electron-phonon interactions could also be important. We argue here that simply using the available electronic structure information for MA TBG, and without any prejudice about the interactions responsible for $\mathrm{SC}$, we can put strong constraints on its superconducting $T_{c}$.

There are two bands for each of the two valleys, one above and the other below the charge neutrality point (CNP). Each band has a twofold spin degeneracy, with bands for one valley related to those of the other by time reversal. We include these eight bands in the $\sum_{m m^{\prime}, \sigma}$ in Eq. (2), while the $\sum_{\mathbf{k}}$ is over the moiré Brillouin zone, a hexagon with side $2 K \sin (\theta / 2) \simeq K \theta$. We use the tightbinding model of Ref. [17], a multiparameter fit to the continuum dispersion [15], to calculate $M_{m, m^{\prime}}^{-1}(\mathbf{k})$ of Eq. (5), which is block diagonal in the valley index, so that there are no cross-valley terms in Eq. (2).

To derive a general bound, where we make no simplifying assumptions, we start with $\tilde{D} \geq 0$ and obtain $\tilde{D} \leq$ $\left(\hbar^{2} / 4 \Omega\right) \sum_{\mathbf{k} m m^{\prime} \sigma}\left|M_{m m^{\prime}}^{-1}(\mathbf{k})\right|\left|\left\langle c_{\mathbf{k} m \sigma}^{\dagger} c_{\mathbf{k} m^{\prime} \sigma}\right\rangle\right|$ using the triangle inequality. We next use the Cauchy-Schwarz inequality to obtain $\left|\left\langle c_{\mathbf{k} m \sigma}^{\dagger} c_{\mathbf{k} m^{\prime} \sigma}\right\rangle\right|^{2} \leq n_{m \sigma}(\mathbf{k}) n_{m^{\prime} \sigma}(\mathbf{k}) \leq 1$, since the momentum distribution $n_{m \sigma}(\mathbf{k}) \leq 1$. We thus find $\tilde{D} \leq\left(\hbar^{2} / 4 \Omega\right) \sum_{\mathbf{k}, m, m^{\prime} \sigma}\left|M_{m m^{\prime}}^{-1}(\mathbf{k})\right|$, which leads to the bound $k_{B} T_{c} \leq 56 \mathrm{~K}$.

We can obtain a more stringent $T_{c}$ bound if we use further physical inputs. The Mott gap in the correlated insulator is experimentally [3,4] known to be $\approx 0.3 \mathrm{meV}$, and we expect a superconducting gap which is at most that value. Thus we may assume that, at half filling away from CNP on the hole-doped side, say, the bands above the CNP are essentially empty and unaffected by pairing.

Before proceeding, we derive a general result valid for arbitrary interactions which shows that interband terms do not contribute to Eq. (2) for completely filled or empty bands. To prove this, we again use the Cauchy-Schwarz inequality $\left|\left\langle c_{\mathbf{k} m \sigma}^{\dagger} c_{\mathbf{k} m^{\prime} \sigma}\right\rangle\right|^{2} \leq n_{m \sigma}(\mathbf{k}) n_{m^{\prime} \sigma}(\mathbf{k})=0$ when either band $m$ or $m^{\prime}$ is empty. A similar argument works for the filled case after a particle-hole transformation; see Appendix E. Thus, $\left\langle c_{\mathbf{k} m \sigma}^{\dagger} c_{\mathbf{k} m^{\prime} \sigma}\right\rangle=0$ for $m \neq m^{\prime}$, whenever either of the two bands is completely filled or empty, and only $m=m^{\prime}$ terms survive in Eq. (2).

To bound $T_{c}$ for MA TBG near half filling on the holedoped side of the CNP, we take $n_{m}(\mathbf{k})=0$ for the empty bands above the CNP, as explained above. Keeping only band-diagonal terms and using the triangle inequality, we obtain $\tilde{D} \leq\left(\hbar^{2} / 4 \Omega\right) \sum_{\mathbf{k}, m, \sigma}\left|M_{m m}^{-1}(\mathbf{k})\right| n_{m \sigma}(\mathbf{k})$. Using $n(\mathbf{k}) \leq 1$ for the bands below CNP we obtain the bound $T_{c} \leq 14.4 \mathrm{~K}$ near half filling for hole doping using the tight-binding model of Ref. [17]. A similar calculation leads to $T_{c} \leq 15.0 \mathrm{~K}$ near half filling for electron doping; see Appendix F. We note that using $\left|M^{-1}\right|$ and general constraints on $n(\mathbf{k})$ leads to rigorous results, but weakens the bounds.

Finally, we make a physically motivated estimate of $\tilde{D}$, which yields an improved, but approximate, result. We use the $T=0$ band theory result $\left\langle c_{\mathbf{k} m \sigma}^{\dagger} c_{\mathbf{k} m^{\prime} \sigma}\right\rangle=$ $\delta_{m, m^{\prime}} \Theta\left(\mu-\epsilon_{m}(\mathbf{k})\right)$, with the chemical potential $\mu$ determined by the density $\Omega^{-1} \sum_{\mathbf{k}, m, \sigma} n_{m \sigma}(\mathbf{k})$. This, together with $M_{m m}^{-1}(\mathbf{k})$ calculated from the tight-binding model of Ref. [17], leads to the density-dependent estimate of $\tilde{D}$ plotted in Fig. 1. We note that using $\partial^{2} / \partial k_{x}^{2}$ versus $\partial^{2} / \partial k_{y}^{2}$ to calculate $M^{-1}$ affects our estimates by less than a percent.

The integrated optical spectral weight, given by $\left(2 \pi e^{2} / \hbar^{2}\right) \tilde{D}$, vanishes at the band insulators when all bands are either filled or empty. Clearly our bandstructure-based estimate does not know about the Mott insulating states at half filling away from CNP. $(\pi / 2)$ times

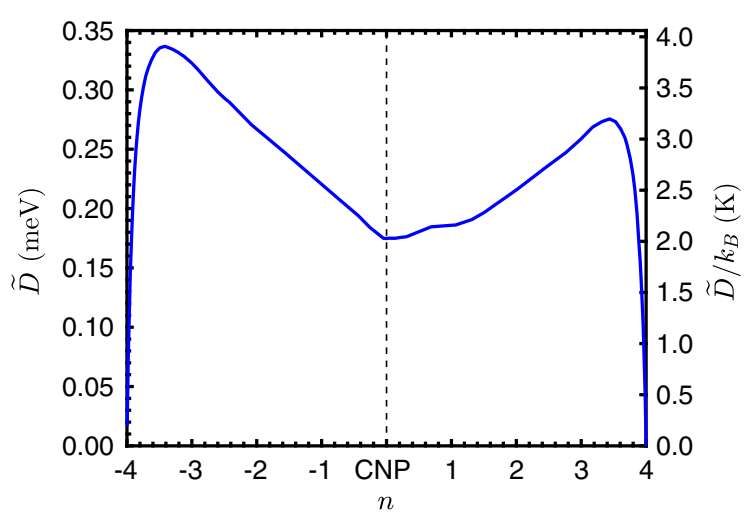

FIG. 1. $\tilde{D}$ as a function of doping from the charge neutrality point (CNP) in magic-angle twisted bilayer graphene (MA TBG), calculated using the band structure of Ref. [17] at $T=0$. $\left(2 \pi e^{2} / \hbar^{2}\right) \tilde{D}$ is the integrated optical spectral weight and $\pi \tilde{D} / 2$ is an upper bound on the SC $T_{c}$ in MA TBG. 
the $\tilde{D}$ plotted in Fig. 1 is an estimated upper bound on the $\mathrm{SC} T_{c}$. The system is not SC over most of the doping range, but our bound is the maximum attainable $T_{c}$ if the system were to exhibit superconductivity. We find the maximum $T_{c}$ to be about $6 \mathrm{~K}$, while the experimental value is $3 \mathrm{~K}$ [29].

We note that the $T_{c}$ bounds are sensitive to the precise electronic structure results we use as input for calculating $M^{-1}$. As shown in Appendix $\mathrm{F}$, using the tight-binding results of Ref. [18] for MA TBG leads to a $T_{c}$ estimate about 2.5 times higher than the one presented above, based on the band structure of Ref. [17]. We emphasize that these differences arise from the fact that the details of the noninteracting band structure of MA TBG are not very well established. Irrespective of that, our results suggest that MA TBG is a strongly correlated SC in a phase fluctuation dominated regime.

\section{2D ATTRACTIVE HUBBARD MODEL AND OPTICAL LATTICES}

We next obtain important insights on the value of $T_{c}$ and its interaction dependence for the 2D attractive Hubbard model, where we can compare our bound with signproblem-free quantum Monte Carlo (QMC) simulations [30]. This system has also been investigated in recent optical lattice experiments [12].

Consider NN hopping on a square lattice with $\mathcal{H}=$ $-t \sum_{\langle i, j\rangle \sigma} c_{i, \sigma}^{\dagger} c_{j \sigma}+$ H.c. $-|U| \sum_{i}\left(n_{i \uparrow}-1 / 2\right)\left(n_{i \downarrow}-1 / 2\right)$. For $n \neq 1$, the system has an $s$-wave SC ground state, exhibiting a crossover from a weak coupling BCS state $(|U| / t \ll 1)$ to a BEC of hard-core on-site bosons $(|U| / t \gg 1)$. The QMC estimate [30] of $T_{c}$, obtained from the BKT jump in the $D_{s}$, is a nonmonotonic function of $|U| / t$ at a fixed density $n$; see Fig. 2 . The BCS mean field

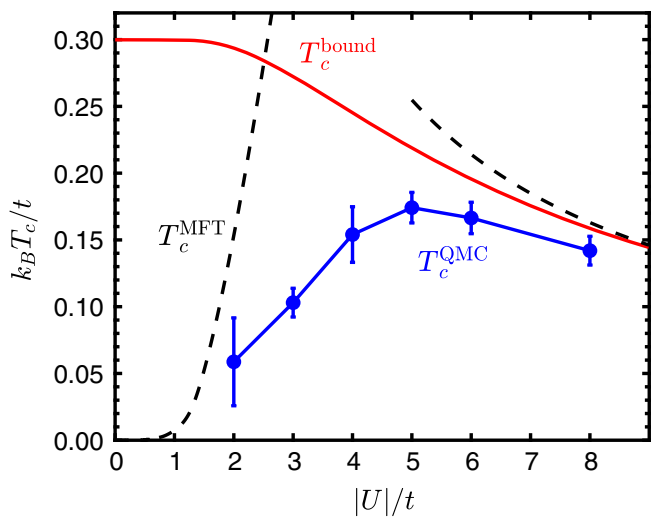

FIG. 2. $T_{c}$ for the $2 \mathrm{D}$ attractive Hubbard model at density $n=$ 0.7 with QMC results from Ref. [30]. The BCS mean-field $T_{c}^{\mathrm{MFT}}$ controls $T_{c}$ at weak coupling. Phase fluctuations, estimated using our upper bound $T_{c}^{\text {bound }}$ (see text), dominate at intermediate and strong coupling, where we also show the $t^{2} /|U|$ asymptotics of our bound.
$T_{c}^{\mathrm{MFT}}$ correctly describes the weak-coupling $T_{c}$. (For a more accurate estimate, one should take into account the GorkovMelik-Barkhudarov correction [25], which suppresses the numerical prefactor but does not alter the functional form of $T_{c}^{\mathrm{MFT}}$.) For $|U| / t>2, T_{c}^{\mathrm{MFT}}$ is the scale at which pairs dissociate and lies well above $T_{c}$. In the $|U| / t \gg 1$ limit we see $T_{c} \sim t^{2} /|U|$, the effective boson hopping.

Our bound permits us to understand $T_{c}(|U| / t)$ in the intermediate coupling regime where there are no other reliable analytical estimates. To estimate $\tilde{D}$ analytically, we need to make an approximation for $n(\mathbf{k})$. If we choose a step function (as we did for the MATBG), we get $T_{c} \leq 0.3 t$ for $n=0.7$, independent of $|U| / t$.

To obtain a better estimate, we note that, as $|U| / t$ increases, the pair size shrinks and $n(\mathbf{k})$ broadens. In the extreme $|U| / t$ limit of on-site bosons, $n(\mathbf{k})$ is flat (k independent), leading to $\tilde{D} \rightarrow 0$, since $\partial^{2} \epsilon / \partial k_{x}^{2}$ is a periodic function with zero mean whose $\mathbf{k}$ sum vanishes. To model this broadening of $n(\mathbf{k})$, we use the results of the $T=0$ BCS-Leggett crossover theory; see Appendix G. This gives us the (approximate) bound plotted in Fig. 2, which has the correct $t^{2} /|U|$ asymptotic behavior at large $|U|$.

In general, we see that $T_{c} \leq \min \left\{T_{c}^{\mathrm{MFT}}, \pi \tilde{D} / 2 k_{B}\right\}$. For temperatures between the pairing scale $T_{c}^{\mathrm{MFT}}$ and $T_{c}$ at which phase coherence sets in, the "normal state" exhibits a pseudogap due to preformed pairs $[13,14]$.

\section{THREE-DIMENSIONAL SYSTEMS}

Experiments suggest that there may be an upper bound on $T_{c}$ in 3D systems; see, e.g., Fig. 6 of Ref. [4]. We have not succeeded in deriving a rigorous bound on the $3 \mathrm{D} T_{c}$, unlike in 2D. There are two challenges that one faces in trying to derive a bound in 3D: one related to rigorous control on numerical prefactors and the other to the functional form of the relation between $T_{c}$ and $D_{s}$. Both are related to the fact that in $3 \mathrm{D}$ the superfluid stiffness does not have dimensions of energy, unlike in 2D.

Following Emery and Kivelson [2], we focus on the 3D phase-ordering temperature $k_{B} T_{\theta}=A D_{s}(0) \bar{a}$, which could provide a bound on $T_{c}$. Here $A$ is a (dimensionless) constant and $\bar{a}$ is the length scale up to which one has to coarse grain to derive an effective XY model. Emery and Kivelson use $\bar{a}^{2}=\pi \xi^{2}$, where $\xi$ is the coherence length, and suggest, based on Monte Carlo results for classical $\mathrm{XY}$ models, that $A \simeq 4.4$ gave a reasonable account of experiments on underdoped cuprates and other materials.

However, the coefficient $A$ is nonuniversal and can vary from one system to another. Consider the $3 \mathrm{D}$ problem of the BCS-BEC crossover in ultracold Fermi gases [10] with $\hbar^{2} k^{2} / 2 m$ dispersion and interaction, characterized by the $s$-wave scattering length $a_{s}$, tuned using a Feshbach resonance. At unitarity $\left(\left|a_{s}\right|=\infty\right)$, the experimental $k_{B} T_{c} \simeq 0.17 E_{F}$ [11], while QMC estimates [31,32] range 
from $k_{B} T_{c} \simeq 0.15 E_{F}$ to $0.17 E_{F}$. QMC estimates show the expected nonmonotonic behavior of $k_{B} T_{c} / E_{F}$ as a function of $1 / k_{F} a_{s}$, with a maximum $k_{B} T_{c} / E_{F} \simeq 0.22$ at a small positive $1 / k_{F} a_{s}$. The maximum value of $k_{B} T_{c} / E_{F}$ is larger than the noninteracting BEC result, consistent with the rigorous result [33] that repulsive interactions increase the $T_{c}$ of a dilute Bose gas in 3D.

We choose $\xi \simeq k_{F}^{-1}$ near unitarity [34] and try to use $k_{B} T_{\theta}=A\left(\hbar^{2} n / 4 m\right)(\sqrt{\pi} \xi)$ as a bound on $T_{c}$. Consistency with the observed $k_{B} T_{c} / E_{F} \simeq 0.22$ then requires $A \simeq 7.4$, quite different from the 4.4 quoted above. We do not know if there is a definite value of $A$ that would give a "phase-ordering" upper bound on $T_{c}$ in 3D.

The following argument suggests that there may, in fact, be no general bound on $T_{c}$ that is linear in $D_{s}(0)$ in 3D. From a practical point of view, one is interested in learning about the highest $T_{c}$ in a class of materials. But, if a general bound were to exist, it should be equally valid in situations where both $T_{c}$ and $D_{s}(0)$ are driven to zero by tuning a (dimensionless) parameter $\delta \rightarrow 0^{+}$toward a quantum critical point (QCP). From the action $S=$ $\frac{1}{2} D_{s} \int_{0}^{\beta} d \tau \int d^{d} \mathbf{r}|\nabla \theta|^{2}+\cdots$ describing the phase fluctuations of the SC order parameter, we get the quantum Josephson scaling relation [35] $D_{s}(0) \sim \delta^{(z+d-2) \nu}$. One also obtains, as usual, $T_{c} \sim \delta^{z \nu}$, where $z$ and $\nu$ are the dynamical and correlation length exponents in $d$ spatial dimensions. Thus, $T_{c} \sim\left[D_{s}(0)\right]^{z /(z+d-2)}$ near the QCP. In 2D, this gives a linear scaling between $T_{c}$ and $D_{s}(0)$. However, in 3D we get $T_{c} \sim D_{s}(0)^{z /(z+1)}$ which, sufficiently close to the QCP, will necessarily violate an upper bound on $T_{c}$ that is conjectured to scale linearly with $D_{s}(0)$. This is not just an academic issue, as experiments see precisely such a deviation from linear scaling with $T_{c} \sim \sqrt{D_{s}(0)}$, consistent with $z=1$, both in highly underdoped [36,37] and in highly overdoped $[38,39]$ cuprates.

\section{CONCLUDING REMARKS}

We have thus far ignored disorder. We note that $D_{s}$ of the pure system is necessarily larger than that in the disordered system. This can be seen by generalizing Leggett's bound [40] on the superfluid density (derived in the context of supersolids) to the case of disordered systems [41]. Thus our upper bounds for translationally invariant systems continue to be valid in the presence of disorder, although they can be improved.

Although we have focused on narrow-band and lowdensity systems here, our bounds have also important implications for systems close to insulating states, either correlation driven or disorder driven. In either case, if there is a continuous superconductor-to-insulator transition, the superfluid stiffness will eventually become smaller than the energy gap and control the SC $T_{c}$.

As a design principle, it is interesting to ask if one can have multiband systems where a narrow band has a large energy gap and large "mean-field" $T_{c}$ interacting with a broadband that makes a large contribution to the superfluid stiffness, thus getting the best of both worlds.

\section{ACKNOWLEDGMENTS}

We are grateful to P. Törmä, S. Peotta, and L. Liang for pointing out an error in an earlier version of our paper that led us to the correct multiband result presented here. We thank J. Kang and O. Vafek for providing the tight-binding parameters for Ref. [18]. We thank S. Kivelson and C. Jayaprakash for very useful conversations related to the argument in Appendix H. We acknowledge support from NSF DMR1410364 and the Center for Emergent Materials, an NSF MRSEC, under Grant No. DMR-1420451.

\section{APPENDIX A: LINEAR RESPONSE, $D_{s}$ AND $\tilde{D}$}

Let us consider the general Hamiltonian

$$
\mathcal{H}=\mathcal{H}_{K}+\mathcal{H}_{\text {int }},
$$

where $\mathcal{H}_{\text {int }}$ represents arbitrary interactions, including those that give rise to superconductivity, and $\mathcal{H}_{K}$ is the most general single-particle Hamiltonian for a multiband or multiorbital lattice model:

$$
\mathcal{H}_{K}=\sum_{i \alpha j \beta \sigma} t_{\alpha \beta}\left(\mathbf{r}_{i \alpha}-\mathbf{r}_{j \beta}\right) c_{i \alpha}^{\dagger} c_{j \beta} .
$$

Here $t_{\alpha \beta}\left(\mathbf{r}_{i \alpha}-\mathbf{r}_{j \beta}\right)$ represents the hopping matrix element from orbital $\beta$ in unit cell $j$ to orbital $\alpha$ in unit cell $i$ with $i, j$ spanning all unit cells, including $i=j$. We omit the spin label $\sigma$ only to simplify notation, but we are not ignoring spin, as emphasized by the spin sum. In the presence of an external vector potential $\mathbf{A}$, the hopping picks up the Peierls phase,

$$
\mathcal{H}_{K} \rightarrow \mathcal{H}_{K}=\sum_{\mathbf{R} \mathbf{r}, \alpha \beta \sigma} t_{\alpha \beta}(\mathbf{r}) e^{-i e \mathbf{A}(\mathbf{R}) \cdot \mathbf{r} / \hbar} c_{i \alpha}^{\dagger} c_{j \beta}
$$

where we use the notation $\mathbf{R}=\left(\mathbf{r}_{i \alpha}+\mathbf{r}_{j \beta}\right) / 2$ and $\mathbf{r}=$ $\mathbf{r}_{i \alpha}-\mathbf{r}_{j \beta}$ for simplicity. Since we are eventually interested in the long-wavelength limit $\mathbf{q} \rightarrow 0$, we choose a very slowly varying vector potential and write $\int_{\mathbf{r}_{j \beta}}^{\mathbf{r}_{i \alpha}} \mathbf{A} \cdot d \mathbf{l} \simeq \mathbf{A}(\mathbf{R}) \cdot \mathbf{r}$.

Within linear response theory we can Taylor expand the exponential, retaining terms which are linear (paramagnetic) and quadratic (diamagnetic) in $\mathbf{A}$. We transform to Fourier space using $t_{\alpha \beta}(\mathbf{k})=\sum_{\mathbf{r}} t_{\alpha \beta}(\mathbf{r}) e^{-i \mathbf{k} \cdot \mathbf{r}}$ and $c_{i \alpha}=$ $\Omega^{-1 / 2} \sum_{\mathbf{k}} e^{i \mathbf{k} \cdot \mathbf{r}_{i \alpha}} d_{\mathbf{k} \alpha}$. We can then write the current operator $j_{x}=\delta \mathcal{H}_{K} / \delta A_{x}$ as the sum of the paramagnetic $(P)$ and diamagnetic $(D)$ current operators given by

$$
j_{x}^{P}(\mathbf{q})=\frac{e}{\hbar \Omega} \sum_{\alpha \beta, \mathbf{k} \sigma} \frac{\partial t_{\alpha \beta}(\mathbf{k})}{\partial k_{x}} d_{\mathbf{k}+\mathbf{q} / 2, \alpha}^{\dagger} d_{\mathbf{k}-\mathbf{q} / 2, \beta},
$$




$$
j_{x}^{D}(\mathbf{q})=\frac{e^{2}}{\hbar^{2} \Omega} \sum_{\alpha \beta, \mathbf{k} \sigma} \frac{\partial^{2} t_{\alpha \beta}(\mathbf{k})}{\partial k_{x}^{2}} d_{\mathbf{k} \alpha}^{\dagger} d_{\mathbf{k} \beta} A_{x}(\mathbf{q}),
$$

where we only show the $x$ component for simplicity. Note that the paramagnetic current operator, when transformed to the band basis, will in general have interband matrix elements $[7,8]$. The only property of $j_{x}^{P}(\mathbf{q})$ that we will need to use below, however, is that it is a Hermitian operator; see Eq. (C1).

The superfluid stiffness $D_{s}$ is defined as the static longwavelength limit of the transverse response of the current density $\mathbf{j}$ to a vector potential $\mathbf{A}$,

$$
\begin{aligned}
& \left\langle j_{x}\right\rangle(\mathbf{q}, \omega)=\frac{-4 e^{2}}{\hbar^{2}} D_{s} A_{x}(\mathbf{q}, \omega), \\
& \text { with } \quad q_{x}=0, \quad q_{\perp} \rightarrow 0, \quad \omega=0,
\end{aligned}
$$

and $\perp$ represents the orthogonal directions to $x$. Standard linear response theory leads to the Kubo formula:

$$
D_{s}=\tilde{D}-\frac{\hbar^{2}}{4 e^{2}} \chi_{j_{x} j_{x}}^{\perp}(\mathbf{q} \rightarrow 0, \omega=0),
$$

where the first term is the diamagnetic term, which is of central interest in this work, and the second is the transverse paramagnetic current-current correlation function. We focus on the latter in Appendix $\mathrm{C}$, where we show that $\chi_{j_{x} j_{x}}^{\perp} \geq 0$ at all temperatures.

Here we focus on the first term that can be read off from the form of the diamagnetic current operator. We find it convenient to write it in the band basis as

$$
\tilde{D}=\frac{\hbar^{2}}{4 \Omega} \sum_{m m^{\prime}, \mathbf{k} \sigma} M_{m m^{\prime}}^{-1}(\mathbf{k})\left\langle c_{\mathbf{k} m}^{\dagger} c_{\mathbf{k} m^{\prime}}\right\rangle
$$

with the inverse mass tensor given by

$$
M_{m m^{\prime}}^{-1}(\mathbf{k})=\sum_{\alpha \beta} U_{m, \alpha}^{\dagger}(\mathbf{k}) \frac{\partial^{2} t_{\alpha \beta}(\mathbf{k})}{\partial\left(\hbar k_{x}\right)^{2}} U_{\beta, m^{\prime}}(\mathbf{k}) .
$$

The unitary transformation $U$ that transforms from the orbital to the band basis is defined by

$$
\sum_{\alpha \beta} U_{m, \alpha}^{\dagger}(\mathbf{k}) t_{\alpha \beta}(\mathbf{k}) U_{\alpha, m^{\prime}}(\mathbf{k})=\epsilon_{m}(\mathbf{k}) \delta_{m, m^{\prime}} .
$$

This allows us to write the final result in the band basis using

$$
d_{\mathbf{k} \alpha}=\sum_{m} U_{\alpha, m}(\mathbf{k}) c_{\mathbf{k} m}
$$

We note several important points about the inverse mass tensor $M_{m m^{\prime}}^{-1}(\mathbf{k})$. (i) It depends only on the bare band structure, and is independent of temperature and interactions, (ii) it has both diagonal and off-diagonal terms in the band indices. and (iii) it is not simply related to the curvature of the bands $\partial^{2} \epsilon_{m}(\mathbf{k}) / \partial k_{x}^{2}$, in contrast to the single-band case in Eq. (A12).

The standard reference on the formalism for calculating the superfluid stiffness in lattice systems is Scalapino, White, and Zhang (SWZ) [21]. Our normalization conventions differ from them and, more importantly, they focus on the special case of a single-band model with nearest-neighbor hopping on a square (or cubic) lattice. Thus it may be useful for us to provide a "dictionary" relating our results to theirs.

In the single-band case our expression for $\tilde{D}$ reduces to

$$
\tilde{D}=\frac{1}{4 \Omega} \sum_{\mathbf{k} \sigma} \frac{\partial^{2} \epsilon(\mathbf{k})}{\partial k_{x}^{2}} n(\mathbf{k}),
$$

where the momentum distribution

$$
n(\mathbf{k})=\left\langle c_{\mathbf{k}}^{\dagger} c_{\mathbf{k}}\right\rangle .
$$

This result is valid for arbitrary one-band dispersion. For the special case of NN hopping on a square (or cubic) lattice, it is easy to see that the right-hand side of Eq. (A12) is proportional to the kinetic energy in the $x$ direction, $\left\langle-K_{x}\right\rangle$ in the notation of SWZ. Our result thus reduces to

$$
\tilde{D} \rightarrow\left\langle-K_{x}\right\rangle / 4 .
$$

Finally, we note that our superfluid stiffness $D_{s}$ is related to that of SWZ by

$$
D_{s}=\left(\hbar^{2} / 4 \pi e^{2}\right) D_{s}^{\mathrm{SWZ}} .
$$

\section{APPENDIX B: RELATION BETWEEN $\tilde{D}$ AND OPTICAL SPECTRAL WEIGHT}

To see that $\tilde{D}$ is proportional to the optical sum rule spectral weight, we identify the dynamical conductivity $\sigma(\omega)$ as the current response to an electric field $\mathbf{E}=-\partial_{t} \mathbf{A}$ :

$$
i \omega \sigma(\omega)=\left[\chi_{j_{x} j_{x}}(\mathbf{q}=0, \omega)-\frac{4 e^{2}}{\hbar^{2}} \tilde{D}\right] .
$$

Using the Kramers-Krönig relation,

$$
\omega \operatorname{Im} \sigma(\omega)=-\frac{2}{\pi} P \int_{0}^{\infty} d \omega^{\prime} \operatorname{Re} \sigma\left(\omega^{\prime}\right) \frac{\omega^{2}}{\omega^{\prime 2}-\omega^{2}},
$$

and $\operatorname{Re} \chi_{j_{x} j_{x}}(\omega \rightarrow \infty) \rightarrow 0$, we obtain the sum rule for the optical conductivity as

$$
\int_{0}^{\infty} d \omega \operatorname{Re} \sigma(\omega)=\frac{2 \pi e^{2}}{\hbar^{2}} \tilde{D}
$$




\section{APPENDIX C: DERIVATION OF BOUND $D_{s} \leq \tilde{D}$}

We show that $\chi_{j_{x} j_{x}}(\mathbf{q}, \omega=0) \geq 0$ at any temperature. This follows directly from its Lehmann representation:

$$
\frac{1}{Z} \sum_{i j}\left[\frac{e^{-\beta E_{i}}-e^{-\beta E_{j}}}{E_{j}-E_{i}}\right]\left|\left\langle i\left|j_{x}^{P}(\mathbf{q})\right| j\right\rangle\right|^{2} \geq 0,
$$

where $|i\rangle$ and $|j\rangle$ are exact eigenstates of the full Hamiltonian $\mathcal{H}$ in Eq. (A1) with eigenvalues $E_{i}, E_{j}$ and $Z=\operatorname{Tr}\left[e^{-\beta \mathcal{H}}\right]$. The last inequality follows from $\left(e^{-x}-e^{-y}\right) /$ $(y-x) \geq 0$. At zero temperature, this expression reduces to

$$
\chi_{j_{x} j_{x}}(\mathbf{q}, \omega=0)=2 \sum_{i} \frac{\left|\left\langle i\left|j_{x}^{P}(\mathbf{q})\right| 0\right\rangle\right|^{2}}{E_{i}-E_{0}} \geq 0,
$$

where $|0\rangle$ is the ground state. From Eq. (A7), we thus conclude that

$$
D_{s} \leq \tilde{D}
$$

\section{APPENDIX D: REAL SPACE BOUND ON $\tilde{D}$}

Except in the case of a single parabolic band, $\tilde{D}$ depends in general on both the $T$ and the interactions, since the thermal average in $\left\langle c_{\mathbf{k} m}^{\dagger} c_{\mathbf{k} m^{\prime}}\right\rangle$ is calculated using the full $\mathcal{H}$. It is thus illuminating to derive an upper bound for $\tilde{D}$ which shows that $\tilde{D}$ must become small when the densities are low or if all the hopping parameters are small. Such a bound for the single-band case with arbitrary dispersion was sketched in the paper. Here we turn to the multiband case.

It is convenient to start with the real space representation:

$$
\tilde{D}=\frac{1}{4 \Omega} \sum_{\mathbf{R r}, \alpha \beta \sigma} r_{x}^{2} t_{\alpha \beta}(\mathbf{r})\left\langle c_{i \alpha}^{\dagger} c_{j \beta}\right\rangle
$$

Here, both forward and backward hopping are accounted for in $\sum_{\mathbf{r}}$ with $t_{\alpha \beta}(|\mathbf{r}|)=t_{\beta \alpha}^{*}(|\mathbf{r}|)$. Since $\tilde{D} \geq 0$, we can use the triangle inequality. Further using the Cauchy-Schwarz inequality we get

$$
\begin{aligned}
\tilde{D} & \leq \frac{1}{4 \Omega} \sum_{\mathbf{R} \mathbf{r}, \alpha \beta \sigma} r_{x}^{2}\left|t_{\alpha \beta}(\mathbf{r})\left\langle c_{i \alpha}^{\dagger} c_{j \beta}\right\rangle\right| \\
& \leq \frac{1}{4 \Omega} \sum_{\mathbf{R} \mathbf{r}, \alpha \beta \sigma} r_{x}^{2}\left|t_{\alpha \beta}(\mathbf{r})\right| \sqrt{n_{i \alpha} n_{j \beta}},
\end{aligned}
$$

where $n_{i \alpha}=\left\langle c_{i \alpha}^{\dagger} c_{i \alpha}\right\rangle$.

Here and below we define an inner product for operators $A, B$ in terms of the thermal expectation value $\left\langle A^{\dagger} B\right\rangle$, which allows us to use the Cauchy-Schwarz inequality $\left|\left\langle A^{\dagger} B\right\rangle\right|^{2} \leq\left\langle A^{\dagger} A\right\rangle\left\langle B^{\dagger} B\right\rangle$.

\section{APPENDIX E: INTERBAND CONTRIBUTIONS TO $\tilde{D}$}

We discuss here the conditions under which we can ignore the interband contributions to $\tilde{D}$ given by

$$
\tilde{D}=\frac{\hbar^{2}}{4 \Omega} \sum_{m m^{\prime}, \mathbf{k} \sigma} M_{m m^{\prime}}^{-1}(\mathbf{k})\left\langle c_{\mathbf{k} m}^{\dagger} c_{\mathbf{k} m^{\prime}}\right\rangle
$$

This requires us to understand when $\left\langle c_{\mathbf{k} m}^{\dagger} c_{\mathbf{k} m^{\prime}}\right\rangle=0$ for $m \neq m^{\prime}$. We show here that this is the case, independent of interactions, when (a) either one of the two bands in empty and (b) when either one of the two bands is fully filled.

We use the Cauchy-Schwarz inequality (see end of Appendix D) to obtain

$$
\left|\left\langle c_{\mathbf{k} m}^{\dagger} c_{\mathbf{k} m^{\prime}}\right\rangle\right| \leq \sqrt{n_{m}(\mathbf{k}) n_{m^{\prime}}(\mathbf{k})}
$$

where $n_{m}(\mathbf{k})=\left\langle c_{m \mathbf{k}}^{\dagger} c_{m \mathbf{k}}\right\rangle$ is the momentum distribution function, and equality holds for $m=m^{\prime}$. For $m \neq m^{\prime}$, if either band is completely empty, $n_{m}(\mathbf{k})=0$ for all $\mathbf{k}$ and the interband contribution to $\tilde{D}$ in Eq. (E1) vanishes.

A similar argument for completely filled bands follows from a particle-hole transformation $c_{m \mathbf{k}} \rightarrow h_{m \mathbf{k}}^{\dagger}$. Since $\left\langle c_{\mathbf{k} m}^{\dagger} c_{\mathbf{k} m^{\prime}}\right\rangle=-\left\langle h_{\mathbf{k} m}^{\dagger} h_{\mathbf{k} m^{\prime}}\right\rangle$,

$$
\begin{aligned}
\left|\left\langle c_{\mathbf{k} m}^{\dagger} c_{\mathbf{k} m^{\prime}}\right\rangle\right| & =\left|\left\langle h_{\mathbf{k} m}^{\dagger} h_{\mathbf{k} m^{\prime}}\right\rangle\right| \\
& \leq \sqrt{n_{m}^{h}(\mathbf{k}) n_{m^{\prime}}^{h}(\mathbf{k})} \\
& =\sqrt{\left[1-n_{m}(\mathbf{k})\right]\left[1-n_{m^{\prime}}(\mathbf{k})\right]} .
\end{aligned}
$$

Thus we conclude that for filled and empty bands, the interband terms do not contribute to the sum in Eq. (E1), even in the presence of arbitrary interactions.

Finally, we note the simple fact that within band theory there are no interband contributions to $\tilde{D}$. In the absence of interactions (denoted by subscript 0 ), we obtain

$$
\left\langle c_{\mathbf{k} m}^{\dagger} c_{\mathbf{k} m^{\prime}}\right\rangle_{0}=f\left(\epsilon_{m}(\mathbf{k})\right) \delta_{m, m^{\prime}},
$$

where $f$ is the Fermi function.

\section{APPENDIX F: MAGIC-ANGLE TWISTED BILAYER GRAPHENE}

Magic angles in twisted bilayer graphene were first predicted by the continuum model [15]. Following up on the experimental discovery of correlation-induced insulators and superconductivity in MA TBG, there has been considerable progress in understanding its electronic structure [17-19]. We first focus on the bounds that we obtain from the tight-binding model of Koshino et al. [17], and then at the end of the appendix we compare these with the 


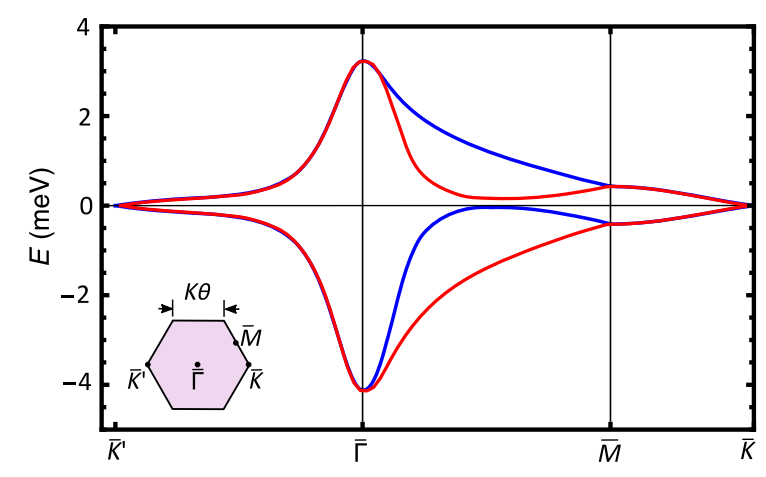

FIG. 3. Energy dispersion for MA TBG along high-symmetry lines in the moiré Brillouin zone (BZ) for the continuum model dispersion [15] that is accurately described by the tight-binding model of Koshino et al. [17]. The bands shown in red and blue correspond to the two valleys of the original $\mathrm{BZ}$ and are related by time reversal.

results we obtain from the tight-binding model of Kang and Vafek [18].

The continuum model dispersion [15] is accurately reproduced by the multiparameter tight-binding fit of Koshino et al. [17] (see Fig. 3), which takes into account hopping over distances up to $9\left|\mathbf{L}_{\mathbf{M}}\right|$, where $\mathbf{L}_{\mathbf{M}}$ is the moiré lattice vector. We use the hopping integrals presented in the Supplemental Material of Ref. [17] to construct the noninteracting Hamiltonian $\mathcal{H}_{K}$ of Eq. (A2). We then identify the unitary matrix $U(\mathbf{k})$ that diagonalizes $t_{\alpha \beta}(\mathbf{k})$ [see Eq. (A10)] and use it together with $t_{\alpha \beta}(\mathbf{k})$ to compute the inverse mass tensor:

$$
M_{m m^{\prime}, a}^{-1}(\mathbf{k})=\sum_{\alpha \beta} U_{m, \alpha}^{\dagger}(\mathbf{k}) \frac{\partial^{2} t_{\alpha \beta}(\mathbf{k})}{\partial\left(\hbar k_{a}\right)^{2}} U_{\beta, m^{\prime}}(\mathbf{k}) .
$$

Note that we have made explicit here the direction $a=x, y$ as an additional subscript on $M^{-1}$.

The inverse mass tensor, obtained from the band-structure information as described above, is used to compute $\tilde{D}_{x}$ and $\tilde{D}_{y}$ and bound $T_{c}$ as described in the paper. The additional input needed to determine $\tilde{D}$ using Eq. (A8) is $\left\langle c_{\mathbf{k} m}^{\dagger} c_{\mathbf{k} m^{\prime}}\right\rangle$, and we took two different approaches to compute this.

In the first approach, we looked at SC near half filling on the hole-doped side of the CNP, and argued that the chemical potential was sufficiently far from the CNP that we can take the band above the CNP to be empty. Then using the result of Appendix $\mathrm{E}$ we can ignore all interband terms with $m \neq m^{\prime}$. For the occupied band we used only the general constraint that $n(\mathbf{k}) \leq 1$. Using the triangle inequality, we then obtain

$$
\tilde{D}_{a} \leq \frac{\hbar^{2}}{4 \Omega} \sum_{\mathbf{k} m, \sigma}\left|M_{m m, a}^{-1}(\mathbf{k})\right|,
$$

where the empty bands above the CNP are excluded from the sum.

A similar reasoning also works for SC in the vicinity of half filling on the electron-doped side of the CNP, where we need to use the fact that the bands below CNP are filled to eliminate interband terms following Appendix E. We use a particle-hole transformation $c_{m \mathbf{k}} \rightarrow h_{m \mathbf{k}}^{\dagger}$, under which $t_{\alpha \beta}(\mathbf{k}) \rightarrow-t_{\alpha \beta}(\mathbf{k})$ and thus $M^{-1} \rightarrow-M^{-1}$. We write $\tilde{D}$ in terms of the hole momentum distribution functions $n_{m}^{h}(\mathbf{k})=\left\langle h_{m \mathbf{k}}^{\dagger} h_{m \mathbf{k}}\right\rangle$ to get

$$
\tilde{D}_{a}=\frac{\hbar^{2}}{4 \Omega} \sum_{m, \mathbf{k} \sigma} M_{m m, a}^{-1}(\mathbf{k})\left(n_{m}^{h}(\mathbf{k})-1\right) .
$$

We then show that the second term on the right-hand side vanishes as follows:

$$
\begin{aligned}
\sum_{m, \mathbf{k}} M_{m m, a}^{-1}(\mathbf{k}) & =\sum_{\mathbf{k}, \alpha \beta} \frac{\partial^{2} t_{\alpha \beta}(\mathbf{k})}{\partial\left(\hbar k_{a}\right)^{2}} \sum_{m} U_{m, \alpha}^{\dagger}(\mathbf{k}) U_{\beta, m}(\mathbf{k}) \\
& =\sum_{\mathbf{k}, \alpha} \frac{\partial^{2} t_{\alpha \alpha}(\mathbf{k})}{\partial\left(\hbar k_{a}\right)^{2}}=0 .
\end{aligned}
$$

We have first used $\sum_{m} U_{\beta, m}(\mathbf{k}) U_{m, \alpha}^{\dagger}(\mathbf{k})=\delta_{\beta, \alpha}$, which follows from the unitarity of $U$, and then the fact that

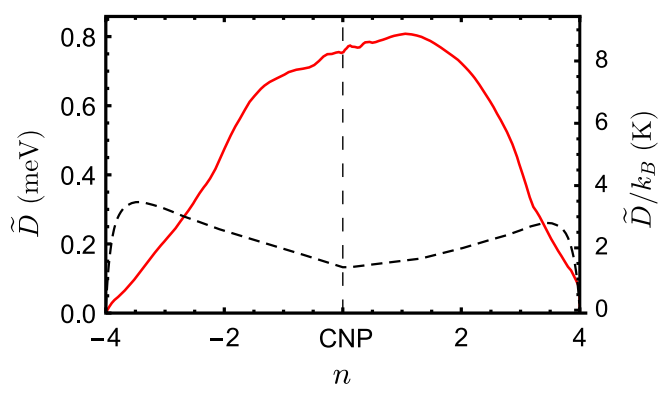

(b)

FIG. 4. Comparison of (a) the band structure and (b) the integrated spectral weight $\tilde{D}$ for the models in Ref. [17] (in black) and Ref. [18] (in red). 
$\partial^{2} t_{\alpha \alpha}(\mathbf{k}) / \partial k_{a}^{2}$ is a periodic function with zero mean, whose $\sum_{\mathbf{k}}$ vanishes. Using the triangle inequality and the general constraint $n^{h}(\mathbf{k}) \leq 1$, we obtain an expression for electron doping which is similar to the hole-doped case:

$$
\tilde{D}_{a} \leq \frac{\hbar^{2}}{4 \Omega} \sum_{\mathbf{k} m, \sigma}\left|M_{m m, a}^{-1}(\mathbf{k})\right|
$$

where now the filled bands below the CNP are excluded from the sum. These bounds, though rigorous, are weak because they involve $\left|M^{-1}\right|$ and only very general constraints on $n(\mathbf{k})$.

The second (approximate) approach was to simply use a $T=0$ (noninteracting) band theory estimate. We thus use Eq. (E4) to obtain

$$
\tilde{D}_{a} \simeq \frac{\hbar^{2}}{4 \Omega} \sum_{\mathbf{k} m, \sigma} M_{m m, a}^{-1}(\mathbf{k}) \Theta\left(\mu-\epsilon_{m}(\mathbf{k})\right),
$$

with the chemical potential $\mu$ determined by the density. We found that $\tilde{D}_{x}$ and $\tilde{D}_{y}$ calculated from the tight-binding model of Ref. [17] differ by less than a percent. The resulting density-dependent $\tilde{D}$ is shown in Fig. 1 of the main paper.

We note that there are many different tight-binding models for describing the narrow bands in MA TBG, and our $T_{c}$ bounds depend on this input. We focused above on the results based on Ref. [17] with an electronic structure that has separate charge conservation at the $K$ and $K^{\prime}$ valleys. A rather different model without valley-charge conservation was derived [18] using only time-reversal and point-group symmetry. We compare in Fig. 4(a) the band structures of Ref. [17] in black and that of Ref. [18] in red. The corresponding integrated spectral weights $\tilde{D}$ are shown in Fig. 4(b) using the same color convention. The maximum $T_{c}$ based on the band structure of Ref. [18] is $15 \mathrm{~K}$, which is 2.5 times larger than that estimated from Ref. [17].

\section{APPENDIX G: ATTRACTIVE HUBBARD MODEL}

It is interesting to ask how our bound on $\mathrm{SC} T_{c}$ in 2D depends on interactions. We use the attractive Hubbard model on a square lattice as a concrete example to understand these trends, and to compare our bound with estimates of $T_{c}$ from sign-problem-free quantum Monte Carlo simulations.

Our bound is $k_{B} T_{c} \leq \pi /(8 \Omega) \sum_{\mathbf{k}, \sigma}\left[\partial_{k_{x}}^{2} \epsilon(\mathbf{k})\right] n_{\sigma}(\mathbf{k})$. This result can be written in terms of the kinetic energy $\left\langle-K_{x}\right\rangle$, as discussed at the end of Appendix A. The interaction dependence is contained in the momentum distribution function $n_{\sigma}(\mathbf{k})$ which, as we argue in the paper, must become increasingly broader and flatter as $|U| / t$ increases. In the weak-coupling BCS limit (small $|U| / t), n_{\sigma}(\mathbf{k})$ is almost like the Fermi function at $T=0$, very slightly broadened by the superconductivity. On the other hand, in the extreme BEC limit (large $|U| / t$ ) of nearly on-site bosons, the $n_{\sigma}(\mathbf{k})$ of the constituent fermions is essentially flat.

We model this $|U| / t$ trend in the momentum distribution using the BCS-Leggett crossover theory expression

$$
n_{\sigma}(\mathbf{k})=\frac{1}{2}\left(1-\frac{\epsilon(\mathbf{k})-\mu}{E(\mathbf{k})}\right)
$$

where $E(\mathbf{k})=\sqrt{(\epsilon(\mathbf{k})-\mu)^{2}+\Delta^{2}}$ is the Bogoliubov quasiparticle energy. The chemical potential $\mu$ and the pair potential $\Delta$ are determined self-consistently for a given density $n$ and attraction $|U|$ by solving the $T=0$ gap and number equations:

$$
\begin{gathered}
\frac{1}{|U|}=\frac{1}{\Omega} \sum_{\mathbf{k}, \sigma} \frac{1}{2 E(\mathbf{k})}, \\
n=\frac{1}{\Omega} \sum_{\mathbf{k}, \sigma} n_{\sigma}(\mathbf{k}) .
\end{gathered}
$$

We see from Fig. 2 that the $T_{c}$ obtained from QMC data [30] is always lower than $T_{c}^{\text {bound }}$. Figure 2 also shows that the bound is most useful in the intermediate to strongcoupling regime, and less useful in the weak-coupling regime where $T_{c}$ is, in fact, well described by $T_{c}^{\mathrm{MFT}}$, the pair breaking energy scale.

\section{APPENDIX H: $T_{c}$ BOUNDS IN SPATIALLY ANISOTROPIC SYSTEMS}

We collect here some results on the role of spatial anisotropy focusing mainly on $2 \mathrm{D}$. We note that various quantities that we have considered are different in different directions labeled by $a=x, y$. We have shown that

$$
D_{s, a}(T) \leq \tilde{D}_{a}(T) .
$$

The most conservative bound on $T_{c}$ in $2 \mathrm{D}$ is then

$$
k_{B} T_{c} \leq \frac{\pi}{2} \max \left\{\tilde{D}_{x}, \tilde{D}_{y}\right\}
$$

Clearly this bound is not optimal because we expect $T_{c}$ to go to zero if either $D_{s, x}$ or $D_{s, y}$ goes to zero. Using BKT theory we can show that

$$
k_{B} T_{c}=\frac{\pi}{2}\left[D_{s, x}\left(T_{c}^{-}\right) D_{s, y}\left(T_{c}^{-}\right)\right]^{1 / 2},
$$

which leads to the improved bound 


$$
k_{B} T_{c} \leq \frac{\pi}{2}\left(\tilde{D}_{x} \tilde{D}_{y}\right)^{1 / 2}
$$

To derive Eq. (H3) we start with the free energy for phase fluctuations:

$$
\mathcal{F}=\frac{1}{2} \int d x d y\left[D_{s, x}\left(\partial_{x} \theta\right)^{2}+D_{s, y}\left(\partial_{y} \theta\right)^{2}\right]
$$

We then rescale lengths using $x^{\prime}=\left(D_{0} / D_{s, x}\right)^{1 / 2} x$ and $y^{\prime}=\left(D_{0} / D_{s, y}\right)^{1 / 2} y$, where $D_{0}$ is any convenient energy scale for normalization, to obtain

$\mathcal{F}=\frac{1}{2}\left(D_{s, x} D_{s, y}\right)^{1 / 2} \int d x^{\prime} d y^{\prime}\left[\left(\partial_{x^{\prime}} \theta\right)^{2}+\left(\partial_{y^{\prime}} \theta\right)^{2}\right]$.

This immediately leads to the generalization of the NelsonKosterlitz result in Eq. (H3). We emphasize that the reason this seemingly naive argument works is that the line of fixed points below $T_{c}$ is actually described by a Gaussian theory and the BKT $T_{c}$ is precisely when vortex-antivortex unbinding becomes relevant at a Gaussian fixed point.

[1] Y. J. Uemura et al., Universal Correlations between $T_{c}$ and $n_{s} / m^{*}$ (Carrier Density over Effective Mass) in High- $T_{c}$ Cuprate Superconductors, Phys. Rev. Lett. 62, 2317 (1989).

[2] V. J. Emery and S. A. Kivelson, Importance of Phase Fluctuations in Superconductors with Small Superfluid Density, Nature (London) 374, 434 (1995).

[3] Y. Cao, V. Fatemi, A. Demir, S. Fang, S. L. Tomarken, J. Y. Luo, J. D. Sanchez-Yamagishi, K. Watanabe, T. Taniguchi, E. Kaxiras, R. C. Ashoori, and P. Jarillo-Herrero, Correlated Insulator Behaviour at Half-Filling in Magic-Angle Graphene Superlattices, Nature (London) 556, 80 (2018).

[4] Y. Cao, V. Fatemi, S. Fang, K. Watanabe, T. Taniguchi, E. Kaxiras, and P. Jarillo-Herrero, Unconventional Superconductivity in Magic-Angle Graphene Superlattices, Nature (London) 556, 43 (2018).

[5] N. B. Kopnin, T. T. Heikkilä, and G. E. Volovik, HighTemperature Surface Superconductivity in Topological Flat-Band Systems, Phys. Rev. B 83, 220503(R) (2011).

[6] E. Tang and L. Fu, Strain-Induced Partially Flat Band, Helical Snake States and Interface Superconductivity in Topological Crystalline Insulators, Nat. Phys. 10, 964 (2014).

[7] S. Peotta and P. Törmä, Superfluidity in Topologically Nontrivial Flat Bands, Nat. Commun. 6, 8944 (2015).

[8] L. Liang, T. I. Vanhala, S. Peotta, T. Siro, A. Harju, and P. Törmä, Band Geometry, Berry Curvature, and Superfluid Weight, Phys. Rev. B 95, 024515 (2017).

[9] W. Ketterle and M. W. Zwierlein, in Making, Probing and Understanding Ultracold Fermi Gases, Proceedings of the International School of Physics "Enrico Fermi," Course CLXIV, edited by M. Inguscio, W. Ketterle, and C. Salomon (IOS Press, Amsterdam, 2008).
[10] M. Randeria and E. Taylor, Crossover from BardeenCooper-Schrieffer to Bose-Einstein Condensation and the Unitary Fermi Gas, Annu. Rev. Condens. Matter Phys. 5, 209 (2014).

[11] M. J. H. Ku, A. T. Sommer, L. W. Cheuk, and M. W. Zwierlein, Revealing the Superfluid Lambda Transition in the Universal Thermodynamics of a Unitary Fermi Gas, Science 335, 563 (2012).

[12] D. Mitra, P. Brown, E. Guardado-Sanchez, S. S. Kondov, T. Devakul, D. A. Huse, P. Schauss, and W. Bakr, Quantum Gas Microscopy of an Attractive Fermi Hubbard System, Nat. Phys. 14, 173 (2018).

[13] M. Randeria, N. Trivedi, A. Moreo, and R. T. Scalettar, Pairing and Spin Gap in the Normal State of Short Coherence Length Superconductors, Phys. Rev. Lett. 69, 2001 (1992).

[14] N. Trivedi and M. Randeria, Deviations from Fermi-Liquid Behavior above $T_{c}$ in $2 D$ Short Coherence Length Superconductors, Phys. Rev. Lett. 75, 312 (1995).

[15] R. Bistritzer and A. H. MacDonald, Moire Bands in Twisted Double-Layer Graphene, Proc. Natl. Acad. Sci. U.S.A. 108, 12233 (2011).

[16] J. M. B. Lopes dos Santos, N. M. R. Peres, and A. H. Castro Neto, Graphene Bilayer with a Twist: Electronic Structure, Phys. Rev. Lett. 99, 256802 (2007).

[17] M. Koshino, N. F. Q. Yuan, T. Koretsune, M. Ochi, K. Kuroki, and L. Fu, Maximally Localized Wannier Orbitals and the Extended Hubbard Model for Twisted Bilayer Graphene, Phys. Rev. X 8, 031087 (2018).

[18] J. Kang and O. Vafek, Symmetry, Maximally Localized Wannier States, and a Low-Energy Model for Twisted Bilayer Graphene Narrow Bands, Phys. Rev. X 8, 031088 (2018).

[19] H. C. Po, L. Zou, A. Vishwanath, and T. Senthil, Origin of Mott Insulating Behavior and Superconductivity in Twisted Bilayer Graphene, Phys. Rev. X 8, 031089 (2018).

[20] G. Baym, The Microscopic Description of Superfluidity, in Mathematical Methods in Solid State and Superfluid Theory, edited by R. C. Clark and G. H. Derrick (Springer, New York, 1968).

[21] D. J. Scalapino, S. R. White, and S. Zhang, Insulator, Metal, or Superconductor: The Criteria, Phys. Rev. B 47, 7995 (1993).

[22] D. R. Nelson and J. M. Kosterlitz, Universal Jump in the Superfluid Density of Two-Dimensional Superfluids, Phys. Rev. Lett. 39, 1201 (1977).

[23] B. Valenzuela, M. J. Calderón, G. León, and E. Bascones, Optical Conductivity and Raman Scattering of Iron Superconductors, Phys. Rev. B 87, 075136 (2013).

[24] M. Randeria, J.-M. Duan, and L.-Y. Shieh, Bound States, Cooper Pairing, and Bose Condensation in Two Dimensions, Phys. Rev. Lett. 62, 981 (1989).

[25] L. P. Gor'kov and T. K. Melik-Barkhudarov, Contribution to the Theory of Superfluidity in an Imperfect Fermi Gas, Zh. Eksp. Teor. Fiz. 40, 1452 (1961) [Sov. Phys. JETP, 13, 1018 (1961)].

[26] D. S. Petrov, M. A. Baranov, and G. V. Shlyapnikov, Superfluid Transition in Quasi-Two-Dimensional Fermi Gases, Phys. Rev. A 67, 031601(R) (2003).

[27] D. S. Fisher and P. C. Hohenberg, Dilute Bose Gas in Two Dimensions, Phys. Rev. B 37, 4936 (1988). 
[28] M. G. Ries, A. N. Wenz, G. Zürn, L. Bayha, I. Boettcher, D. Kedar, P. A. Murthy, M. Neidig, T. Lompe, and S. Jochim, Observation of Pair Condensation in the Quasi-2D BEC-BCS Crossover, Phys. Rev. Lett. 114, 230401 (2015).

[29] M. Yankowitz, S. Chen, H. Polshyn, Y. Zhang, K. Watanabe, T. Taniguchi, D. Graf, A. F. Young, and C. R. Dean, Tuning Superconductivity in Twisted Bilayer Graphene, Science 363, 1059 (2019).

[30] T. Paiva, R. Scalettar, M. Randeria, and N. Trivedi, Fermions in 2D Optical Lattices: Temperature and Entropy Scales for Observing Antiferromagnetism and Superfluidity, Phys. Rev. Lett. 104, 066406 (2010).

[31] E. Burovski, E. Kozik, N. Prokof'ev, B. Svistunov, and M. Troyer, Critical Temperature Curve in BEC-BCS Crossover, Phys. Rev. Lett. 101, 090402 (2008).

[32] O. Goulko and M. Wingate, Thermodynamics of Balanced and Sightly Spin-Imbalanced Fermi Gases at Unitarity, Phys. Rev. A 82, 053621 (2010).

[33] R. Seiringer and D. Ueltschi, Rigorous Upper Bound on the Critical Temperature of Dilute Bose Gases, Phys. Rev. B 80, 014502 (2009).

[34] J. R. Engelbrecht, M. Randeria, and C. A. R. Sáde Melo, BCS to Bose Crossover: Broken-Symmetry State, Phys. Rev. B 55, 15153 (1997).
[35] M. P. A. Fisher, P. B. Weichman, G. Grinstein, and D. S. Fisher, Boson Localization and the Superfluid-Insulator Transition, Phys. Rev. B 40, 546 (1989).

[36] I. Hetel, T. R. Lemberger, and M. Randeria, Quantum Critical Behaviour in the Superfluid Density of Strongly Underdoped Ultrathin Copper Oxide Films, Nat. Phys. 3, 700 (2007).

[37] D. M. Broun, W. A. Huttema, P. J. Turner, S. Özcan, B. Morgan, Ruixing Liang, W. N. Hardy, and D. A. Bonn, Superfluid Density in a Highly Underdoped $\mathrm{YBa}_{2} \mathrm{Cu}_{3} \mathrm{O}_{6+\mathrm{y}}$ Superconductor, Phys. Rev. Lett. 99, 237003 (2007).

[38] T. R. Lemberger, I. Hetel, A. Tsukada, M. Naito, and M. Randeria, Superconductor-to-Metal Quantum Phase Transition in Overdoped $\mathrm{La}_{2-\mathrm{x}} \mathrm{Sr}_{\mathrm{x}} \mathrm{CuO}_{4}$, Phys. Rev. B 83, 140507(R) (2011).

[39] I. Božović, X. He, J. Wu, and A. T. Bollinger, Dependence of the Critical Temperature in Overdoped Copper Oxides on Superfluid Density, Nature (London) 536, 309 (2016).

[40] A. J. Leggett, Can a Solid be "Superfluid"?, Phys. Rev. Lett. 25, 1543 (1970).

[41] A. Paramekanti, N. Trivedi, and M. Randeria, Upper Bounds on the Superfluid Stiffness of Disordered Systems, Phys. Rev. B 57, 11639 (1998). 